\title{
SPORT PSYCHOLOGY IN THE PARALYMPIC ENVIRONMENT: PERCEPTIONS OF COACHES, ATHLETES AND MANAGERS
}

\author{
Alan RINGLAND ${ }^{1 *}$ \\ ${ }^{1}$ Institute of Technology, Health \& Leisure Department, Dromtacker, Tralee, Ireland \\ *Corresponding author: alan.ringland@staff.ittralee.ie
}

https://doi.org/10.35189/dpeskj.2020.59.1.2.

\begin{abstract}
While there have been significant benefits from the delivery of sport psychology services to Paralympic athletes, there is dearth of research into the dimension of performance within the Paralympic environment. This investigation looked at the perceptions of the significant others in the sporting environment, such as performance managers, coaches, support staff and organisations. Following semi-structured interviews with 10 members of the Irish Paralympic team including athletes (3), coaches (2), individual sport managers (2), sports science and medical staff (1), support staff (1) and the performance manager (1), 5 themes related to their retrospective reflections of the London 2012 Paralympic Games were identified. These general dimensions and higher-order themes included: Communications (top down, timing, lack of communication), Expectation (home games, personal, family, sports council/organisational), Stress (personal, social, cultural, uncertainty), Interpersonal (relationships, stigma, cohesion, power influence) and Environmental (roles, expertise in coaching, diversity of ability, support). The confidentiality of the interview process provided a more honest interpretation of what feedback could be utilised for the next Paralympic cycle. Statements were both positive and negative but expressed in a constructive manner where athletes, coaches, management and support staff recognised the significance of working in an all-inclusive and rounded method to develop and promote a better Team culture and future success at major Games.
\end{abstract}

Keywords: organisational stress, Paralympic, performance environment.

\section{Introduction}

For most athletes, the Olympic and Paralympic Games represent the highpoint of their sporting achievement and career. Winning gold medals are typically recognised as the ultimate accolade of a successful athletic career and arguably, the most demanding challenge an athlete can pursue (Arnold \& Sarkar, 2015; Sarkar \& Fletcher, 2014; Greenleaf, Gould \& Dieffenbach, 2001). This is, in part, due to the timing and emblematic nature of the quadrennial Olympic and Paralympic Games. This infrequency, combined with the magnitude, public media and the worldwide popularity of the sport industry, ensures global interest in and fascination with sporting and athletic performance (Sarkar \& Fletcher, 2014). This concentrated scrutiny, however, brings with it huge pressure and only those who can manage the stress that accompanies sport at this level will be successful. It is no surprise, then, that there has been an increased effort to understand the most effective ways to prepare athletes and teams for Olympic competition (see e.g. Gould \& Maynard, 2009). As the opportunities for sport participation have increased for individuals with disabilities so has the level of competition. Athletes with disabilities now routinely compete at the national and international level with participation in the Paralympic Games as the crowning opportunity. It is now widely accepted that the quality and standards at the Paralympic Games are ever increasing and getting closer to their able-bodied counterparts. In the 100 metres T13 
category, Jason Smyth ran a 10.46 in comparison with a 9.63 ran by Usain Bolt in respective Paralympic and Olympic finals. Another example would be in the T34 1,500 metres in which Rheed McCracken's 3:30.12 gets ever closer to Noah Neary's 3:32.07. However, for toplevel athletes competing with disabilities, research indicates their needs and experiences are more similar to able-bodied elite athletes than different (Dieffenbach \& Statler, 2012; Martin 2012; Ringland, 2013). In the context of sport, the competition is to better recognise, reproduce and sustain world-class performances. Investigating methods to understand the most effective ways to prepare athletes and teams for major competitions has evolved from a number of sources from performance leadership and management to anecdotal reports from successful athletes and coaches (Arnold \& Sarkar, 2015; Fletcher \& Arnold, 2011; Gould \& Maynard, 2009; Olusoga et al., 2012). Athletic performance is multifactorial. A holistic approach to the athletic profile advocates many factors required for world-class sporting performance, yet key personal, lifestyle, relational, training and performance practices can sometimes be overlooked, especially in the performance leadership literature (Arnold \& Sarkar, 2015; Hanton, Fletcher, \& Coughlan, 2005). Empirical research has rarely considered the interacting and over reaching concepts involved in the process of trying to attain exceptional standards in sports, as it has mainly focused on one main domain without promoting all the facets and supporting variables that interact and produce successful and sometimes disappointment (Sarkar \& Fletcher, 2014; Olusoga et al., 2012). Hence, a more holistic approach maybe more beneficial in developing an empirical model where practitioners can have access to and utilise a more all-inclusive evidence-base. The growing body of literature concerning organisational stress suggests that it may be a critical factor in determining well-being and performance development in sport (Burns, Weissensteiner \& Cohen, 2018; Arnold \& Fletcher, 2012). The highly complex social and organisational environment of elite sport imposes numerous demands on the performers and personnel that function within it (i.e., preparation, expectations, interpersonal relationships), with advice frequently sought from psychologists on dealing with the pressures that accompany participation and engagement in the performance process (Mellalieu et al., 2007; Mellalieu \& Shearer, 2013). However, in many instances National Governing Bodies (NGB) have been inadequately and only reactive both recognizing and effectively addressing such issues (Fletcher \& Hanton, 2003; Woodman \& Hardy, 2001). This is in sharp contrast to the widespread competitive and performance stress research (Devonport, 2015; Woodman \& Hardy, 2001; Hanton et al., 2005). Little is known about athletes' involvements of stress, related to the organisational environment within which they operate, despite the fact elite athletes experience more demands associated with the sport organisation than with competitive performance (Arnold \& Sarkar, 2015; Hanton, Fletcher, \& Coughlan, 2005; Melllieu et al., 2009). Research highlights that sports organisations promote a highly demanding environment, with the unique demands (stressors) faced by performers emanating from a range of sources and categorised into many higher order themes such as:

- Personal;

- Organisational;

- Environmental;

- Leadership; 
- Interpersonal;

- Team.

This can lead to strain and negative performance consequences (Burns et al., 2018; Pain \& Harwood, 2008; Mellalieu et al., 2009; Woodman \& Hardy, 2001).

Personal criteria have comprised of injury, expectations, nutrition and well-being (Ringland, 2018), while environmental themes have included selection, finances, training and accommodation (Woodman \& Hardy, 2001) and performance issues have included preparation, technical, tactical psychological and physical (Mellalieu et al., 2009). Interpersonal issues include conflict, social support, building positive and supportive relationships (Burns et al., 2018). Many others overlap including the Team and Leadership themes that centre on coaching, styles, roles, communication and managing (Arnold \& Sarkar, 2015; Burns et al., 2018; Mellialieu et al., 2009; Woodman \& Hardy, 2001). In particular, conflict within the organisational environment of sport is a significant source of strain, with interpersonal conflict among athletes, coaches, and management a salient factor (Mellalieu \& Shearer, 2013; Olusoga et al., 2012). Conflict is a complex construct that can occur at many levels (organisational, group, individual). The outcomes have substantial effects on group function, performance and inter and intra-relationships (Arnold \& Sarkar, 2015; Jehn, 1995). In sport, conflicts exist for many reasons but in the performance environment and at major competitions these tend to relate to interpersonal difficulty between coach, performance director and athlete(s) (Fletcher, Hanton, \& Wagstaff, 2012). Leadership is 'a fundamental aspect of sports performance, particularly within team sport environments' (Cotterill \& Fransen, 2016) and this has encompassed many facets from coaching and leadership to styles and skills as well as including Team issues within the theme (Hanton et al., 2005). Within the organisational stress literature, the raw data or quotations 'speak for themselves', which facilitates the readers' understanding (Woodman \& Hardy, 2001). However, the interpretation of the data may not be contextually or culturally part of the sport organisation. The stressor may yield positive as well as negative outcomes, explanations, and deductions from contextual and directional parameters need examining before drawing conclusions on raw data.

The purpose of this research is to provide a multi-personnel perspective from a unique Paralympic environment. The objective is to offer an informative outlook from a purposive sample of practitioners working in the Paralympic context. The qualitative interpretations of personnel working and competing in the London 2012 Paralympic Games explored the perspectives of participants, the meaning they gave to their experiences.

\section{Methods}

The Paralympic team (2012) were contacted and asked to discuss their perceptions of the London Games. It was explained that they would have to partake in interviews that would cover their sporting experience in the 2012 Paralympic Games. What were the key factors to which Team Ireland attributed their success was the primary goal; with how we could improve in the next cycle. 5 coaches, 22 athletes, 3 sport managers, 6 sports science and medical and 10 support staff replied. The author attempted to select participants who had different experiences and roles of the performance environment and sporting organisation. 
Changes in personnel, throughout the entire team increase the likelihood of a change in the organisational climate (Woodman \& Hardy, 2001). Consequently, in order to set the present study within a relatively short period, the author decided to include only those team members who were still involved in international competition. Open-ended, semi structured face-toface interviews were conducted with Paralympic athletes (3), coaches (2), individual sport managers (2), sports science and medical staff (1), support staff (1) and the performance manager (1). The sample comprised of 7 males and 3 females who ranged in age between 20 and $56(\mathrm{M}=38.2, \mathrm{SD}=10.63)$. The sports represented were Boccia, Athletics, Equestrian and Cycling. Ethics were approved by Paralympics Ireland. A thematic approach guided by grounded theory principles to source and analyse their perspectives and experiences and determine common themes was employed. Mutual times for the interviews were then agreed upon and informed consent attained before data collection. In keeping with ethical procedures regarding confidentiality, all 10 participants were given a code (e.g., Athlete 1, Coach 1). A semi-structured interview guide was developed to ensure that all 10 participants were asked the same set of major questions about their experience of the Games and improvements for the future. The questions focused not only on the day of competition but also about previous indicators of the performance environment. Most of the interview questions were based on the researchers' experience and knowledge of sport organisations. The interview began by thanking them for their participation and then asked about their background and experience. This was followed by: 'I am interested in your experience of the Paralympic Games, the preparation, the future, the positives, the negatives and the environment'. Could you tell me about your experience? Follow-up and elaboration and clarification questions were used throughout the interviews to elicit in-depth information and to ensure that we had discussed everything they felt relevant (Rumbold, Fletcher, \& Daniels, 2018). How did that feel? Did it have an effect? On what exactly? Could you elaborate on that please? Opportunities to provide or to add anything not discussed during the interview was provided. The first author conducted all the interviews. They were carried out either face to face, in person. Each interview lasted approximately 40-60 minutes and was audio recorded. The recorded interviews were transcribed verbatim and analysed. The analysis was both deductive and inductive using axial and open coding (Patton, 2002; Rumbold et al., 2018).

\section{Analysis}

During the initial stages, the first author immersed himself in the data by adopting a reflective approach (Rumbold et al., 2018). This involved listening to each of the interview recordings to gain clarification and reading and re-reading the written transcripts. Following this, segments of quotes that represented similar meanings were inductively coded and grouped into lower-order themes. These lower-order themes were clustered into higher-order themes in the light of established organisational stress frameworks (e.g., Arnold \& Fletcher, 2012; Woodman \& Hardy, 2001). The analysis was deductive in that the overall areas of the study were delineated by the literature and interview guide (e.g., role, training, environment) and axial coding was used to find evidence of the persons' roles and practices. 


\section{Results}

The results that emerged from the data analysis represent the Paralympic participants' combined responses. From the interview data, 189 raw quotes were extracted, which were grouped into 19 lower-order themes. These themes were grouped together into 5 higher-order dimensions. Five dominant and overlapping themes emerged:

Table 1. Participants' Experiences of Competing/Working in the Paralympic Games

\begin{tabular}{lll}
\hline Frequency & \multicolumn{1}{c}{ Lower Order } & Higher Dimensions \\
\hline$(23)$ & Top down, Timing, Leadership & Communications \\
$(77)$ & Home games, Personal, Family, Sports council/organisational & Expectation \\
$(26)$ & Personal, Social, Cultural, Uncertainty & Stress \\
$(53)$ & Relationships, Stigma, Cohesion, Power influence & Interpersonal \\
$(10)$ & Roles, Expertise in coaching, Diversity of ability, Support & Environmental \\
\hline
\end{tabular}

\section{Communication}

Many organisations have top-down communication, but the timing in particular alienated staff and inefficient information channels at the games was handled poorly, as many staff commented,

'I was always last to know about arrangements...' and an athlete remarked 'We were never told or asked what was required and this leads to frustration and then problems...' (A1).

Others felt that 'it is important that we talk to each other to enhance all our performances, coaches, athletes, support staff...' while a coach stated that 'It is always best to take total control in the big events, it makes the athletes feel we know what we are doing...' (C2).

Another said that 'At the Games, I was always told what to do and when to do it and this attitude made me feel angry.... as I like to plan and communicate with my athlete..'(C1).

Communication takes many forms in sport and within a multisport environment best practice may be best shared, as a sports science, and medical staff commented,

'We worked well at home in our team weekend camps; it was easy to see over the past few years how much the group had developed. Perhaps it was the stress of the event and the importance but the reason we did work so well was because we had an open forum at these meetings prior to London....we were able to express ideas to one another, such as in a conversation or debate' (SSM1).

Viewing the group in isolation when looking at performance environments is a mistake. An all-inclusive understanding of leadership in the team environment that accounts for managers, coaches, and support staff is required prior to any event. Exploring team leadership at this level with provide a more realistic picture of the leadership needs, frameworks, and roles at play.

\section{Expectation}

This dimension portrayed the highest number of responses and while linked with Stress as a dimension, the raw data within the context of the conversation and the narrative at the time 
was recalled. One athlete (A2) stated that she 'had set realistic goals, knew what to expect in London and was going to do better than what others hoped', while another relished the event by saying that 'It was my first Paralympics and I was always searching for outcome and perfection...It was a great experience' (A3).

Another athlete (A3) mentioned in the early stages of competition that "everyone I had talked to in my whole village had bought a ticket and I had not even reached a qualification standard'. This did cause the athlete personal anxiety and stress. The sports science and medical provider remarked that "many comments on the table constantly went on about how many people were routing for them and they hoped that they did not let them down" (SS2). He felt that information should be transferred to the sport psychologist to help them deal with their overpowering circumstance.

The Performance Manger felt that "Beijing was a good Team performance with 5 medals, but London was our 'home' games as we could never stage an event this big....so performance was all that mattered...' and this was further evidenced by a manger 'You are only judged by your performance. It is all that matters".

The performance culture depends upon the group, the approach and the management of organisations, teams and individuals. A high-performance culture drives the sports organisation, defines its actions and carves its road to success. This culture may influence positively across all capacities.

"I am the favourite, people expect me to win and break records. That is okay. I am used to it. I welcome it" (A1).

As one athlete stated, being a favourite is not a concern as adopting a personal approach to illustrate the advantages of being the best helped him derive confidence and attract recognition. A manager commented "that expectations and realities were illustrated the last night of the holding camp when after the affirmations of what we have achieved and the team support structure portrayed by video and pictures...the quote that really stood out for me was individual PB's and medals were something to be gained rather than lost" (M2).

This culture of a group and their desire and direction forward should be encouraged. However, negatives can derive from what has happened previously in a group and one needs to be aware of the context.

A sports manager expressed dissatisfaction with this attitude by "All (Sports organisation) they are concerned about is medals. No respect for the individuals, the work they have put in to get here, the sacrifices and time and money they have spent. Let them compete but also enjoy and savour the event as it is a wonderful experience and a lifelong ambition for all of them" (M1).

\section{Stress}

'I was not sure what to expect. I performed well all season. I did well in the Worlds, but everything about London seemed to be about performance and medals. I was lucky not to have been injured like many of my competitors. I had never been to the Paralympics. All this attention scared me' (A2).

The athlete was enjoying competition, but this was a daunting experience. The media attention, the sports gear, the holding camp made her nervous because it was not normally her 
routine. All the support staff there to help was terrifying as it was the uncertainty and the apprehension of a different event.

One of the mangers had real concerns about the safety and the environment that London evoked. 'It was the ease to which athletes and people could readily saunter into London, use the tube, walk the shops in Westfield and just become a tourist. It was not like any other Paralympics' (M1).

This was also exemplified by the support staff's comment about the opening ceremony. 'Here I was, all dressed up and waiting patiently with ... when I noticed that Iran and Iraq were in front of me and Israel directly behind me. This could be a war zone ...'. While there was less cultural uncertainty than being in Beijing there was doubt and uncertainty amongst staff as one manger commented: 'At least you know what type of food is available and edible'(SS2).

\section{Interpersonal}

'The whole team knew each other better than ever before. In Beijing, I knew the staff in my sport and not really any of the others until the village and then it is show time. This time I made an effort to meet, help and make friends. We were together, the weekends, the holding camp, everything. We were made for London 2012' (A2).

Building up relationships and facilitating positive relationships and providing the opportunities for inter-personal relationships that allow athletes to laugh and have time out from the pressures of competition were particularly valued and came in many forms including with friends and team-mates or with support staff and sports science and medical personnel who expand on a purely technical or professional relationship.

'I remember our psychologist at a test event in London. There was a possible bomb scare and he joked about being used to it and then proceeded to tell one of his jokes. I remember some of it, but it had us all in fits of laughter and all the other teams wanted a part of it. They could not believe that in a time of crisis we were laughing'.

This athlete also remarked on the fact that 'within the team, one can sense a hierarchy of ability amongst us the disabled. It is sort of sick in a weird way' (A1).

While there are positive outcomes in the interpersonal category, there were also negative ones. One coach was particularly frustrated at the village where his plans constantly changed, and his wishes ignored. 'It seemed to me that all our efforts to get here were going to be pointless unless we took control and stop the hierarchy of officialdom in the group' (C2).

When asked to elaborate, he highlighted 'that routines, plans, nutrition, sleep patterns, training schedules were being ignored to take in the event, the atmosphere... and that certain parts of the management were preventing him from access'.

One manager stated that the 'Holding camp and preparation was great to know each other and bond. But in the village that changed and the climate we had in Portugal had evaporated due to certain people' (M2).

When management elaborated about anything that may have disrupted performance in the village one said that 'Conflict will always emerge in stressful situations; it was a case of managing it accordingly'. 
When asked if there was a protocol for people disrupting performance, the performance manager said 'Of course and we always managed people before and after their events. It was our responsibility as members of the team to respect everyone. We changed rooms, apartments and outside personnel to accommodate and we anticipated these incidents'.

Another remark illustrated that 'If our coach had not provoked and stirred other members of the Irish team, I am sure we would have performed better...'

Conflict is a natural process of managing people, and an effective sports organisation must respond to conflict and control it. Intragroup conflict is inevitable. Managing intragroup conflict and the effect on individual and team performance is an area that sport psychologists and personnel should lead and direct.

\section{Environmental}

One member of the support team was overwhelmed with the Paralympics when saying that 'It was an interesting experience for me as I had never seen such ability in those with disability... I would never have believed it watching riders with no legs control and perform on a horse' (SS1).

A manager concluded that the team 'had performed so well without funding that the Paralympic organisation should take note that it was the athletes and their social support group that achieved these medals, not the Sports Council' (M2).

One of the coaches stated that 'it was the best and most effective sports science and medical team they had ever had and why was it not available every event'. The support network was positive. This was not the case in funding as it concerned all personnel.

'I was away now for 12 weeks in 2012. I really enjoy it but am always feel a little short changed. Athletes get grants. Other personnel do it as their full-time job. My role here has changed from not only looking after O... but also others in the apartment and at the training...I am not enumerated for any of this and I thought this is the place to say it' (SS2).

'Promoting athletic growth and development, well-being, and elite performance is my goal' (C2), while one of the support staff (SS1) summed up their role as 'I listen to everyone's voices and engaging in dialogue and try and create a positive environment as well as my normal duties'.

\section{Discussion}

The results illustrate the multidimensional nature of both trying to orchestrate elite performance but also becoming aware of the varying perceptions of each staff category. Collaboration may facilitate a better information exchange within a multifaceted sports environment that may in turn reduce power conflicts and interpersonal dissatisfaction. The National Governing Bodies or Sports agencies directly involved for these sports need to address conflict issues. They are essentially difficult to prescribe or implement, especially in groups that are unwilling to change. Training and recruitment for staff to support, to broaden, to be aware of and to contribute are competencies required for all personnel working in sports organisations. Unfortunately, many are dependent upon volunteerism in the Paralympic sector. 
The communication and interpersonal themes in the performance environment of sport and organisational issues with this sample are interrelated. A wide range of supportive communication channels need implementation but can be at odds with autonomy controlling communication, so a variety of information channels in compliance with the culture of the organisation and of the individuals within the organisation is required. Communication in previous research (Fletcher \& Hanton, 2003; Mellalieu et al., 2009) highlights as a sub-theme but has wider implications if inappropriately controlled. Creating supportive interpersonal relationships is not a passive process, but rather a vibrant interplay, which evolves and grows as the individual moves along their development pathway. This involves dynamic and transactional communication between all providers and personnel. Individuals are not machines. Every person is different and their endeavour to have healthy, socially engaging and rewarding relationships in the performance environment is a worthwhile goal, while also succeeding at their chosen sport. A more integrated approach with a supportive social network is key to building a successful environment for athletes, coaches, managers and support personnel to flourish both on and off the field. Interpersonal relationships that allow athletes to laugh and have time out from the pressures of the high-stakes competition were particularly valued and came in many forms including with friends and teammates or with experts such as masseurs, physiotherapists or coaches, who expand on a purely technical or professional relationship (Burns et al., 2018).

Stress as a higher dimension concerned the welfare of the team with safety being of primary concern. This is contrary to research in the organisational literature. Stress associates with the highs and lows of competition, negative chance events such as injury and illness and the typical demands of high-performance sport for example, travel, funding and non-selection (Arnold, Fletcher, \& Daniels, 2017; Devonport, 2017; Woodman \& Hardy, 2001). A strong social support framework alleviates some of these stressors but is like a scaffold only temporary. Team cohesion featured on 12 occasions within the interpersonal theme. Hence the need to observe organisational stress in a holistic manner centring on the people rather than the outcome. This cohesion counterbalances the negativity of the perceived stressors. The 'price of gold' as one coach remarked, has become part of the sporting organisation culture. High-performance emerges as the umbrella term that captures the elite sports. The catchword concept of high performance however has negatives such as injury, overtraining, burnout, depression and other clinical psychological disorders (Fletcher, Hanton, \& Mellalieu, 2006; Gabbett, 2016; Ringland, 2018). It has become the cultural buzzword of sporting organisations. However, if performance and results or podium positions are all that sport is concerned with, then ethically we fall short of having any humanistic value system (Arnold \& Fletcher, 2012). Cultures and sporting performance management systems are about results, as one of the interviewees stated that 'you are only judged by your performance'. The return for the investment by results sought by the sporting organisations illustrates a lack of humanity and duty of care to all individuals involved, yet it drives performance standards' but also promotes resentment and negativity. Athletic performance, coaching performance, management performance and support teams play a more pivotal role than just getting a gold medal. They enhance the social, emotional and holistic and value driven development of those who engage, interact and support. They play a vital role in determining the well-being of those individuals in the performance environment. Working as 
a multidisciplinary team in sporting organisations such as the Paralympics is an essential critical component of effective and sustained working practice, as well as providing an inclusive positive supporting environment for all personnel in the team. One of the support staff who said her role was 'to listen, to create a positive environment and do her normal duties' exemplified this. One of the limitations is that people 'cannot lift the results of interviewing out of the contexts in which they were gathered and claim them as objective data with no strings attached' (Qu \& Dumay, 2011) and in this environment, neither could they.

In conclusion, better people make a better team. Sport is complex. So are people. Combining the two make it a challenging place to work in but if we listen, communicate, plan, organise and implement then sporting competitions and organisations are a fruitful environment to be part of.

\section{References}

Arnold, R., \& Fletcher, D. (2012). A research synthesis and taxonomic classification of the organizational stressors encountered by sport performers. Journal of Sport and Exercise Psychology, 34(3), 397-429. DOI:n10.1123/jsep.34.3.397

Arnold, R., Fletcher, D., \& Daniels, K. (2017). Organisational stressors, coping, and outcomes in competitive sport. Journal of Sports Sciences, 35(7), 694-703. DOI: 10.1080/02640414.2016.1184299

Arnold, R., \& Sarkar, M. (2015). Preparing athletes and teams for the Olympic Games: Experiences and lessons learned from the world's best sport psychologists. International Journal of Sport and Exercise Psychology, 13(1), 4-20. https://doi.org/10.1080/1612197X.2014.932827

Burns, L., Weissensteiner, J., \& Cohen, M. (2018). Lifestyles and mindsets of Olympic, Paralympic and world champions: is an integrated approach the key to elite performance?

British Journal of Sports Medicine, 53(13). http://dx.doi.org/10.1136/bjsports-2018099217

Cotterill, S., \& Fransen, K. (2016). Athlete leadership in sport teams: Current understanding and future directions. International Review of Sport and Exercise Psychology, 9(1), 116133. DOI: 10.1080/1750984X.2015.1124443

Devonport, T. (2015). Understanding stress and coping among competitive athletes. In A. Lane (Ed.), Sport and exercise psychology topics in applied psychology (pp. 127-154). Hove: Routledge.

Dieffenbach, K., \& Statler, T. (2012). More similar than different: The psychological environment of Paralympic Sport. Journal of Sport Psychology in Action, 3(2), 109-118. https://doi.org/10.1080/21520704.2012.683322

Fletcher, D., \& Arnold, R. (2011). Qualitative study of performance leadership and management in elite sport. Journal of Applied Sport Psychology, 23, 223-242. https://doi.org/10.1080/10413200.2011.559184

Fletcher, D., \& Hanton, S. (2003). Sources of organisational stress in elite sports performers. The Sport Psychologist, 17(2), 175-195. DOI: 10.1123/tsp.17.2.175

Fletcher, D., Hanton, S., \& Mellalieu, S. D. (2006). An organisational stress review: Conceptual and theoretical issues in competitive sport. In S. Hanton \& S. D. Mellalieu (Eds.), Literature reviews in sport psychology (pp. 321-373). Hauppauge, NY: Nova Science Publishers. 
Fletcher, D., \& Hanton, S., \& Wagstaff, C. (2012). Performers' responses to stressors encountered in sport organisations. Journal of Sports Sciences, 30(4), 349-358. doi: $10.1080 / 02640414.2011 .633545$

Gabbett, T. (2016). The training-injury prevention paradox: should athletes be training smarter and harder? British Journal of Sports Medicine, 50, 273-280. http://dx.doi.org/10.1136/bjsports-2015-095788

Gould, D., \& Maynard, I. (2009). Psychological preparation for the Olympic Games. Journal of Sports Sciences, 27(13), 1393-1408. https://doi.org/10.1080/02640410903081845

Greenleaf, C., Gould, D., \& Dieffenbach, K. (2001). Factors influencing Olympic performance: Interviews with Atlanta and Nagano U.S. Olympians. Journal of Applied Sport Psychology, 13(4), 254-184. https://doi.org/10.1080/104132001753149874

Hanton, S., Fletcher D., \& Coughlan G. (2005). Stress in elite sport performers: A comparative study of competitive and organisational stressors. Journal of Sports Sciences, 23(10), 1129-1141. DOI: 10.1080/02640410500131480

Jehn, K. A. (1995). A multimethod examination of the benefits and detriments of intragroup conflict. Administrative Science Quarterly, 40(2), 256-282. DOI: 10.2307/2393638

Martin, J. (2012). Mental preparation for the 2014 Winter Paralympic Games. Clinical Journal of Sport Medicine, 22(1), 70-73. doi: 10.1097/JSM.0b013e31824204cc.

Mellalieu, S., Neil, R., Hanton, S., \& Fletcher, D. (2009). Competition stress in sport performers: Stressors experienced in the competition environment. Journal of Sports Sciences, 27(7), 729-744. DOI: 10.1080/02640410902889834

Mellalieu, S., \& Shearer, D. (2013). A preliminary survey of interpersonal conflict at major games and championships. The Sport Psychologist, 27(2), 120-129. https://doi.org/10.1123/tsp.27.2.120

Olusoga, P., Maynard, I., Butt, J., and Hays, K (2012). Coaching under pressure: Mental skills training for sports coaches. Sport and Exercise Psychology Review, 10(3), 31-44.

Pain, M., \& Harwood, C. (2008) The performance environment of the England youth soccer teams: A qualitative investigation. Journal of Sports Sciences, 26(11), 1157-1169.

Patton, M. Q. (2002). Qualitative research and evaluation methods (3rd ed.). Newbury Park, CA: Sage Publications.

Qu, S., \& Dumay, J. (2011). The qualitative research interview. Qualitative Research in Accounting \& Management, 8(3), 238-264. https://doi.org/10.1108/11766091111162070

Ringland, A. (2018). Paralympic experiences. The Division of Sport and Exercise Psychology Annual Conference, 48-49. Retrieved from https://www.bps.org.uk/sites/bps.org.uk/files/Events\%20-

$\% 20$ Files/DSEP2018\%20abstract\%20WEB.pdf

Ringland, A. (2013). A psychological framework for developing success: From 5 to 16 in 4 years. Reflective Practice: International and Multidisciplinary Perspectives, 14(5), 680690. DOI: $10.1080 / 14623943.2013 .839448$

Rumbold, J. L., Fletcher, D., \& Daniels, K. (2018). Using a mixed method audit to inform organisational stress management interventions in sport. Psychology of Sport and Exercise, 35, 27-38. DOI: 10.1016/j.psychsport.2017.10.010

Sarkar, M., \& Fletcher, D. (2014). Psychological resilience in sport performers: a review of stressors and protective factors. Journal of Sports Sciences, 32(15), 1419-1434. doi: $10.1080 / 02640414.2014 .901551$

Woodman, T., \& Hardy, L. (2001). A case study of organisational stress in elite sport. Journal of Applied Sport Psychology, 13, 207-238. doi:10.1080/104132001753149892 